\title{
Circulating angiopoietin-2 is elevated in patients with neuroendocrine tumours and correlates with disease burden and prognosis
}

\author{
R Srirajaskanthan, G Dancey ${ }^{1}$, A Hackshaw ${ }^{2}, T$ Luong $^{3}$, M E Caplin \\ and $T$ Meyer ${ }^{1}$
}

\author{
Neuroendocrine Tumour Unit, Royal Free Hospital, London NW3 2QG, UK \\ ${ }^{1}$ UCL Cancer Institute, University College London, 72 Huntley Street, London WC1E 6BT, UK \\ ${ }^{2}$ Cancer Research UK and UCL Cancer Trials Centre, University College London, London W1T 4TJ, UK \\ ${ }^{3}$ Department of Histopathology, Royal Free Hospital, London NW3 2QG, UK \\ (Correspondence should be addressed to T Meyer; Email: t.meyer@ucl.ac.uk)
}

\begin{abstract}
Angiogenesis is an essential process in the development and growth of tumours. There are a large number of angiogenic mediators including the angiopoietin (Ang) family and vascular endothelial growth factor, which play an important role in both physiological and pathological angiogenesis. This study examines serum levels of Ang-1 and Ang-2 in patients with neuroendocrine tumour (NET) compared healthy controls. ELISA for Ang-1 and Ang-2 was performed in 47 patients with histologically proven NETs and 44 healthy controls. Immunohistochemical staining for Ang-2 was performed in patients to demonstrate cellular location of Ang-2. Serum Ang-2 levels were significantly elevated in patients compared controls (median 4756 vs $2495 \mathrm{pg} / \mathrm{ml}, P<0.001$ ), while there was no significant difference in Ang-1 levels. The ratio of Ang-2:Ang-1 was significantly elevated in patients compared controls $(0.13$ vs $0.066, P<0.001)$. Serum Ang-2 levels were significantly elevated in patients with distant metastases compared with those without metastasis (median 5080 vs $3360 \mathrm{pg} / \mathrm{ml}, P=0.01$ ). There was also a significant increase between Ang-2 levels and volume of liver metastases $(P=0.014)$. Time to disease progression was worse in patients with serum Ang-2 levels $>4756 \mathrm{pg} / \mathrm{ml}(P=0.04)$. Serum Ang-2 but not Ang- 1 is elevated in NET patients. Ang-2 may be a useful serum marker for monitoring and assessment of prognosis in patients with NETs.
\end{abstract}

Endocrine-Related Cancer (2009) 16 967-976

\section{Introduction}

Angiogenesis is an essential process in cancer growth, maintenance and metastasis. It is regulated by the balance between pro- and anti-angiogenic factors (Folkman et al. 1989, Holash et al. 1999). There are a large number of angiogenic mediators including the angiopoietin (Ang) family and vascular endothelial growth factor (VEGF), which play an important role in both physiological and pathological angiogenesis (Holash et al. 1999, Carmeliet \& Jain 2000, Machein et al. 2004). To date, four Ang have been identified; termed Ang 1-4 (Lee et al. 2004). Of these, Ang-1 and Ang-2 are the most widely studied and function as ligands for Tie-2, which is a receptor tyrosine kinase specifically expressed on endothelial cells (Suri et al. 1996, Oliner et al. 2004).

Ang-1 binds specifically to Tie- 2 causing activation by phosphorylation. Ang-1 is produced by endothelial cells and pericytes and is widely expressed in adult tissue, where it appears to have a stabilizing effect on blood vessels (Davis et al. 1996). The role of Ang-1 in tumour development is complex and studies have shown both pro- and anti-angiogenic effects with this growth factor. Ang-2 is expressed at sites of vascular remodelling (Maisonpierre et al. 1997) and promotes vessel destabilization (Yu \& Stamenkovic 2001). 
This appears to be accomplished by Ang- 2 binding to Tie-2 and therefore blocking Ang-1 binding. Ang-2 appears to be a non-signal transducing ligand and therefore disrupts normal Tie-2 activation (Bach et al. 2007). The endothelial cells are then acted upon by various angiogenic factors such as VEGF, which lead to proliferation (Wang et al. 2005).

In tumours, a shift in the balance pro- and antiangiogenic factors is thought to occur; termed the 'angiogenic switch' resulting in an angiogenic phenotype (Folkman \& Hanahan 1991, Tanaka et al. 2003). It has been proposed that a change in the ratio of Ang-1:Ang-2 in favour of Ang-2 might play a role in this switch (Tait \& Jones 2004). Support comes from animal studies in colonic and gastric tumours transfected with Ang-2, which larger and heavier in nude mice compared those transfected with Ang-1 (Ahmad et al. 2001, Etoh et al. 2001).

Neuroendocrine tumours (NETs) are uncommon tumours that can occur in almost any organ and are thought to originate from neuroendocrine cells (Caplin et al. 1998, Modlin et al. 2008). The most common sites of origin is from gastroenteropancreatic tract, where there is a reported incidence of 2-5 per 100000 population (Modlin et al. 2003) but the incidence and prevalence are increasing (Modlin et al. 2003). NETs are generally regarded as slow growing in comparison adenocarcinomas; however, can behave aggressively and often produce highly vascular liver metastases. To date, chromogranin A $(\mathrm{CgA})$ is the best biochemical marker for NETs; however, it lacks specificity and sensitivity especially in patients with low-volume disease (Eriksson et al. 2000, de Herder 2007).

The serum levels of Ang have not been evaluated in NET patients. The aim of this study was to measure serum Ang-1 and Ang-2 levels in patients with NETs and assess their interrelationship and clinical significance.

\section{Materials and methods}

\section{Patients}

We enrolled 47 patients with NETs, between July 2007 and March 2008. All patients had histological confirmation, including assessment of morphology and immunohistochemical analysis for neuron-specific enolase, $\mathrm{CgA}$, synaptophysin and PGP9.5. All cases were well or moderately differentiated and no patients enrolled in the study had poorly differentiated NET. The presence of necrosis, number of mitoses and Ki67 index evaluated. Tumours were graded using the system proposed by European Neuroendocrine
Tumour Society consensus group (Rindi et al. 2007, Eriksson et al. 2008). Using this classification, low-grade tumour was regarded as mitotic count $<2$ per 10 high-power fields (HPF) and Ki67 $\leq 2 \%$, intermediate grade as having a mitotic count 2-20 per $10 \mathrm{HPF}$ and Ki67 3-20\%. All patients underwent imaging with CT or MRI and appropriate nuclear medicine imaging including somatostatin (SST) receptor scintigraphy using ${ }^{111}$ In-DTPA-Phe I-Pentetreotide (Octreoscan, Mallinckrodt Inc, St Louis, MO, USA), within 2 months of blood sampling to enable staging of disease. Serum $\mathrm{CgA}$ was measured in all patients at the same time as serum collection for the study. All previous and current therapies that patients had received were recorded from patient records. The demographics of both groups are shown in Table 1.

Table 1 Demographic data

\begin{tabular}{|c|c|c|}
\hline & NET & Control \\
\hline Subject number & 47 & 44 \\
\hline Age & $61.4(34-80)$ & $59.65(24-86)$ \\
\hline Males & 26 & 23 \\
\hline Females & 21 & 21 \\
\hline \multicolumn{3}{|l|}{ Primary tumour } \\
\hline Bronchial & 3 & \\
\hline Pancreatic & 17 & \\
\hline Jejunal & 2 & \\
\hline Ileal & 20 & \\
\hline Unknown & 5 & \\
\hline \multicolumn{3}{|l|}{ Functional types } \\
\hline Carcinoid syndrome & 18 & \\
\hline Glucagonoma & 1 & \\
\hline Non-functional & 28 & \\
\hline \multicolumn{3}{|l|}{ Histological grade } \\
\hline Low & 24 & \\
\hline Intermediate & 23 & \\
\hline \multicolumn{3}{|l|}{ Biochemical markers } \\
\hline Chromogranin $\mathrm{A}>60$ & 35 & \\
\hline Chromogranin A 0-60 & 12 & \\
\hline $\mathrm{CgA}(\mathrm{pmol} / \mathrm{l})$ (range) & $\begin{array}{l}\text { Median } 99 \\
\quad(17->1000)\end{array}$ & \\
\hline $\begin{array}{l}\text { Concurrent SST } \\
\text { analogues }\end{array}$ & 29 & \\
\hline \multicolumn{3}{|l|}{ Previous therapies } \\
\hline Previous chemotherapy & 4 & \\
\hline Radiotargetted therapy & 4 & \\
\hline Surgery & 19 & \\
\hline
\end{tabular}

All therapies are previous treatments that patients had undergone, except somatostatin (SST) analogues in all patients were currently on at the time of study. Histological grade was classified using the ENETS proposed system for grading (Rindi et al. 2007). The classification was expanded to include bronchial and tumours of unknown primary site. Chromogranin A measurements were made using commercial RIA kit (Roche), which measures the pancreastatin fragment of chromogranin $\mathrm{A}$, normal range is $0-60 \mathrm{pmol} / \mathrm{l}$. 
Survival data and time to progression of disease were identified in all cases. Time to progression was assessed using radiological evidence of disease progression according to RECIST criteria (Therasse et al. 2000), and was calculated in days from time of blood collection. Patients underwent cross-sectional imaging for restaging of their disease on a 3-4 monthly basis.

As control subjects, 44 healthy volunteers who were age and sex matched were enrolled in the same period (Table 1). Most subjects in the control group were healthy relatives of patients and the remainder subjects were volunteers. All control patients had no previous history of cancer.

The study was approved by the Local Ethical Committee and all participants gave written informed consent prior to obtaining samples.

\section{ELISAs}

Serum samples were obtained from each individual, immediately placed on ice and allowed to stand for $30 \mathrm{~min}$. Following this, the samples were centrifuged at $1000 \mathrm{~g}$ for $15 \mathrm{~min}$. Sera were stored at $-80^{\circ} \mathrm{C}$. ELISA were used to measure Ang-1 and Ang-2 (Quantikine, R\&D Systems, Minneapolis, MN, USA). Serum samples were diluted as appropriate prior to being added to separate microplates, each containing a specific antibody for Ang-1 or Ang-2. The mixtures were then incubated for $2 \mathrm{~h}$ on an orbital microplate shaker. Plates were then washed four times to remove unbound antigen. Enzyme-linked polyclonal antibodies specific for each angiogenic factor were then added and the mixture incubated for $2 \mathrm{~h}$. Plates were then washed four times prior to the substrate solution being added to the wells. The colour was allowed to develop following which the stop solution was added. The optical density of each well was determined at $540 \mathrm{~nm}$.

$\mathrm{CgA}$ was measured using a commercial RIA that measures the pancreastatin fragment of $\mathrm{CgA}$.

\section{Immunohistochemical analysis of Ang-2}

Formalin-fixed paraffin-embedded tumour tissues were available from nine patients with a histologically confirmed diagnosis of NET in whom serum samples had been collected for Ang-2 analysis. All cases had been assessed for grade of tumour; including Ki67 proliferation index and number of mitoses per $10 \mathrm{HPF}$ were available in all cases. The study population comprised of six primary pancreatic NETs and three midgut NETs.
Four micrometre sections of tumour tissue were dewaxed three times in xylene and rehydrated in ethanol. Endogenous peroxidase activity was blocked by incubation in $0.5 \%$ hydrogen peroxide, diluted in acetone, for $10 \mathrm{~min}$. Slides were microwaved for $10 \mathrm{~min}$ at 600 in citrate buffer and then transferred to a humidity chamber. Slides were incubated overnight in primary monoclonal Ang-2 antibody at a dilution 1:50 (Santa Cruz product no. Sc-74403). Slides were washed with Post Primary Block (Novolink Max Polymer RE7280 detection kit) for $30 \mathrm{~min}$ in a humidity chamber at room temperature. Slides were washed in TBS-t (Tris buffered saline) and left for 5 min. Novolink Polymer (RE7280-K) was added for $30 \mathrm{~min}$ in a humidity chamber for $30 \mathrm{~min}$, following which slides were washed in TBS-t. The sections were then developed with Novolink 3,3'-diaminobenzidine tetrahydrochloride (DAB) solution for $5 \mathrm{~min}$ by adding $50 \mu \mathrm{l}$ of DAB chromagen to $1 \mathrm{ml}$ of Novolink DAB substrate buffer. The slides are counterstained with Mayer's haematoxylin for $3 \mathrm{~min}$. Positive controls for Ang-2 were renal tissue; negative controls included substitution of the primary antibody with normal sera. An experienced histopathologist ( $\mathrm{T}$ V L) performed the interpretation of immunohistological staining for the antibody studied.

\section{Statistical analysis}

Statistical analysis was performed using GraphPad Prism version 5.00 for Windows (GraphPad Software, San Diego, CA, USA, www.graphpad.com). The values were generally not normally distributed, so non-parametric tests were used to compare marker levels between groups, i.e. the Mann-Whitney $U$ test (for two groups) or Kruskal-Wallis test (for several groups). Multivariate linear regression analyses were used to examine Ang-1 and Ang-2 (on a logarithmic scale) in relation to several other factors. Spearman correlation coefficient was used to analyse correlations between parameters. Time to disease progression curves were plotted using the Kaplan-Meier method and the log-rank test applied.

We also evaluated the screening performance of the markers using the detection rate (sensitivity), defined as the proportion of NET cases that had levels above a specified cut-off, and the false-positive rate, defined as the proportion of controls that had levels above the same cut-off. The likelihood ratio quantifies the 'power' of the marker and is the detection rate divided by the false-positive rate; the higher the value the better the marker. 


\section{Results}

\section{Serum Ang-1 and -2 levels in patients with NETs}

Figure 1 shows scatterplots of the distributions of Ang-1, Ang-2 and the ratio of the two. Patients with NETs had significantly higher serum Ang-2 levels than the control group (median 4756 vs $2495 \mathrm{pg} / \mathrm{ml}$, $P<0.001)$. There was still a highly statistically significant difference when allowing for age and sex $(P<0.001)$. Median serum Ang-1 levels were not different between the two groups $(39135 \mathrm{pg} / \mathrm{ml}$ controls versus $39405 \mathrm{pg} / \mathrm{ml}$ cases; $P=0.60$ ). The ratio of Ang-2 to Ang-1 was significantly increased in patients (median 0.133 compared with 0.066 in controls $P<0.001$ ), but this probably largely reflects the difference associated with Ang-2. There was no evidence of a correlation between serum Ang-2 and Ang-1 $(r=0.11, P=0.49)$; Ang-1 and plasma CgA $(r=-0.22, P=0.13)$; or Ang-2 and plasma CgA $(r=0.03, P=0.8)$. We conducted a multivariate analysis to look at the association between each of Ang-1, Ang-2 and the ratio, and three prognostic factors: histological grade, $\mathrm{CgA}$ and stage. There was some evidence of an association between histological grade and Ang-2, even after allowing for stage and CgA $(P=0.003)$; the geometric mean was 4140 and $6668 \mathrm{pg} / \mathrm{ml}$ in the low and intermediate grades respectively. There was no evidence of an association between either Ang-1 or Ang-2:Ang-1 ratio and the prognostic factors.

To confirm that there was no significant daily variation in Ang-2 levels, we obtained two matched serum samples from 17 control patients separated by $24 \mathrm{~h}$. There was no significant difference between Ang-2 levels $(P=0.143)$ or Ang-1 $(P=0.662)$.

\section{Ang levels and type of NET}

Compared to controls (median $2495 \mathrm{pg} / \mathrm{ml}$ ), serum Ang-2 was significantly elevated in both midgut NETs (median $4790 \mathrm{pg} / \mathrm{ml}, P<0.001$ ) and foregut primary NETs (median $4900 \mathrm{pg} / \mathrm{ml}, \quad P<0.001$ ). When comparing levels between foregut and midgut primary tumours, there was no evidence of a difference in serum Ang-2 (median 4900 vs $4790 \mathrm{pg} / \mathrm{ml}, P=0.59$ ), serum Ang-1 (median 39100 vs $33750 \mathrm{pg} / \mathrm{ml}$, $P=0.28$ ) or the ratio of Ang-2 to Ang-1 (median 0.133 vs $0.147, P=0.68$ ). There was no significant difference in Ang-2 levels between patients with functional tumours $(n=19)$ and non-functional tumours ( $n=28$; median 4503 vs $4867 \mathrm{pg} / \mathrm{ml}$, $P=0.58$ ). Furthermore, there was no difference in serum Ang-2 levels between the 29 patients currently
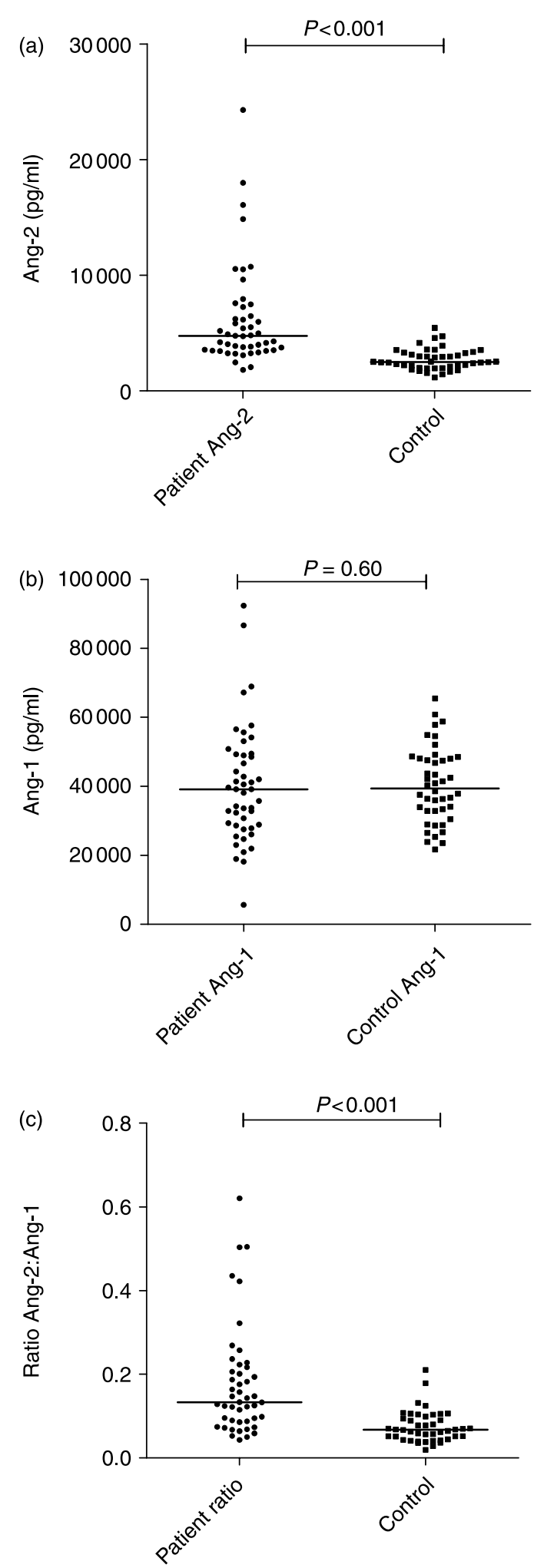

Figure 1 Comparisons of Ang-2, Ang-1 and Ang-2:Ang-1 ratios in patients with NETs $(n=47)$ and controls $(n=44)$. (a) Ang-2 levels, (b) Ang-1 levels and (c) ratio of Ang-2 to Ang-1. 
on SST analogues and 18 patients who were not (median $4755 \mathrm{vs} 4500 \mathrm{pg} / \mathrm{ml}, P=0.82$ ). Similarly for Ang-1 levels (median 40550 vs $33900 \mathrm{pg} / \mathrm{ml}$, $P=0.24)$.

\section{Ang levels and stage of disease}

Ang-2 levels were elevated in patients with metastatic disease compared with those with localized disease without distant metastases (median 5081 vs $3359 \mathrm{pg} / \mathrm{ml}, P=0.01$ ); see Fig. 2. Ang-2 levels were significantly higher in patients with localized nonmetastatic disease $(n=6)$, compared healthy controls (3360 vs $2495 \mathrm{pg} / \mathrm{ml}, P=0.02$; Fig. 3).

From radiological assessment of CT or MRI scans performed within 2 months of blood sampling, visual assessments were made of the volume of liver metastases present. Four categories were created; localized disease without metastases, low-volume liver metastases (involving $<25 \%$ of liver), intermediate(involving $25-50 \%$ of liver) and large-volume liver metastases ( $>50 \%$ of liver), see Fig. 4 . There was a statistically significant difference between the groups when comparing localized disease, low-, intermediateand large-volume liver metastases - $(P=0.014$, one-way ANOVA test), with a suggestion that increased Ang- 2 is associated with increased tumour burden.

\section{Serum Ang-2 as a marker of disease}

Receiver operator curves for serum Ang-2 were constructed to determine the cut-off values for specificity and sensitivity of Ang-2 and Ang-2:Ang-1

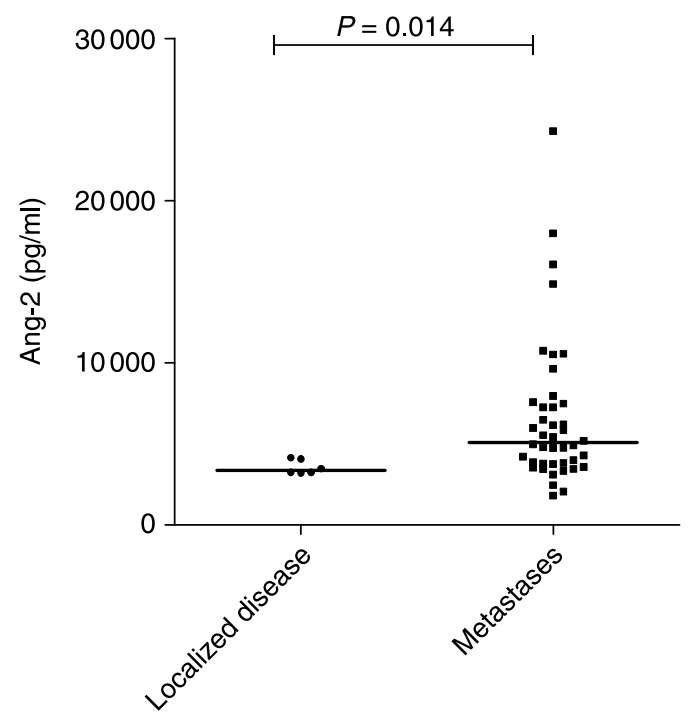

Figure 2 Comparison of serum Ang-2 levels in patients with liver metastases ( $n=41$ cases) and those with localized disease $(n=6)$.

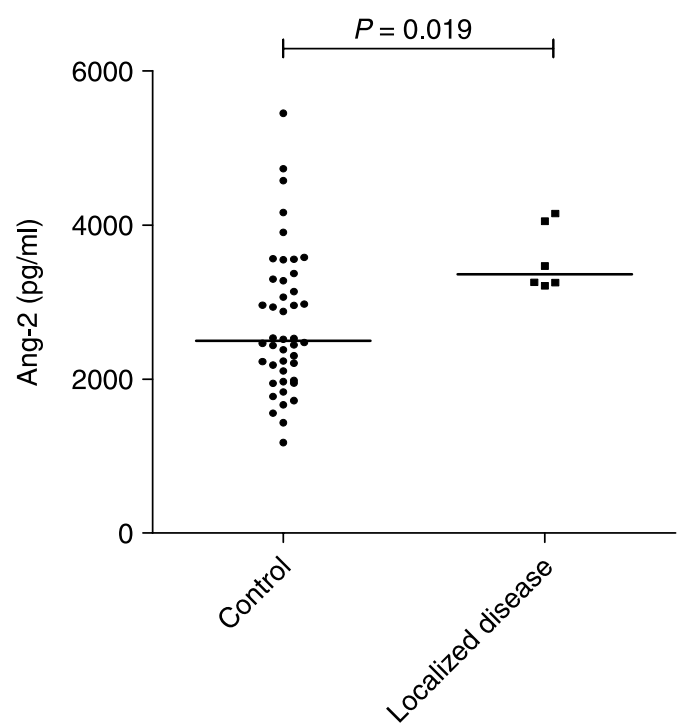

Figure 3 Serum Ang-2 levels are significantly raised in patients with localized non-metastatic disease ( $n=6$ cases) compared healthy controls $(n=44)$.

ratio. The area under the curve for serum Ang-2 was 0.88 and was greater than Ang-1 (0.53; Fig. 5). Detection rate, false-positive rate and likelihood ratio for Ang-2 and the ratio of Ang-2 to Ang-1 are shown in Table 2. Serum Ang-2 was a better marker than the ratio, with a sensitivity of $85 \%$, for a false-positive rate of $22.7 \%$. The $\mathrm{CgA}$ assay has been previously validated and a cut-off of $60 \mathrm{pmol} / \mathrm{ml}$ has been defined. Using this cut-off in our study, $\mathrm{CgA}$ had a sensitivity of $80.9 \%$.

\section{Immunohistochemical staining}

Staining for Ang-2 was identified in five out of the nine tumour samples. In all cases with positive Ang-2 staining, the corresponding serum Ang-2 levels were greater than the control population, see Table 3. Of the four samples that were negative for Ang-2 staining, the serum Ang-2 was raised above the $>4756 \mathrm{pg} / \mathrm{ml}$ (median Ang-2 value for patient cohort) in three cases. The staining pattern was predominantly cytoplasmic with clear tumour staining present. There was no staining of the background liver tissue and staining was localized only to tumour cells and endothelium. In one case, there was strong staining in $10 \%$ of tumour cells metastatic to the gallbladder; however, there was only moderate $1 \%$ staining of the surrounding tumour in liver metastases, see Fig. 6. Ang-2 staining was seen in low- and intermediate-grade tumours as well as pancreatic and midgut tumours. The immunohistochemical studies demonstrate there is intra-tumoural variation in staining patterns for Ang-2. 


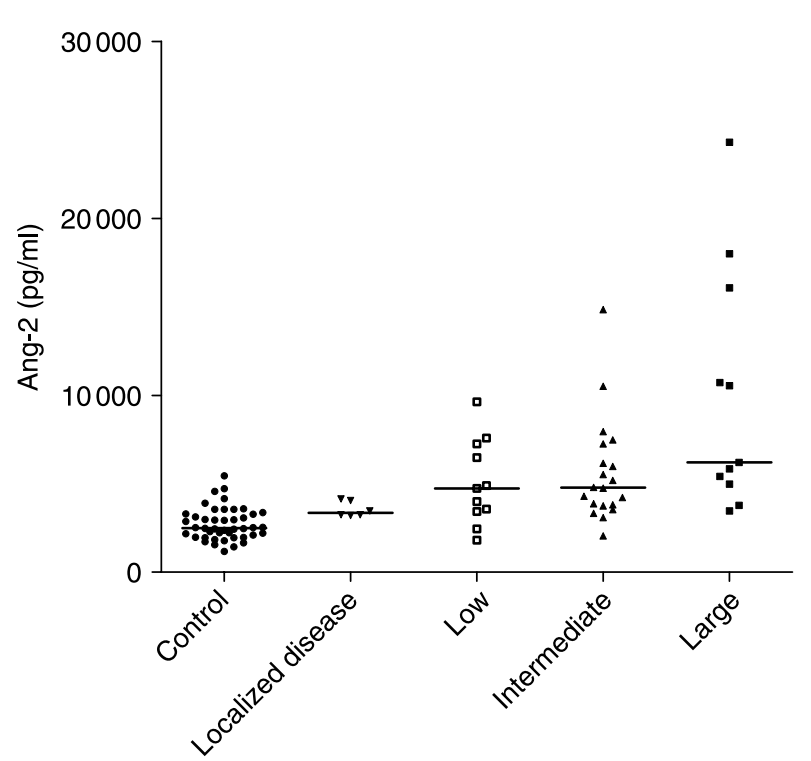

Figure 4 Serum Ang-2 levels in controls $(n=44)$, patients without metastatic disease $(n=6)$ and those with low- $(<25 \%$ liver metastases) $(n=11)$, intermediate- $(25-50 \%$ liver metastases) $(n=19)$ and large-volume ( $>50 \%$ liver metastases) $(n=11)$ liver metastases.

\section{Survival data}

Owing to the indolent nature of these tumours, survival data alone are difficult to obtain (only four patients died during the 8 month follow-up). We therefore examined time to disease progression. Patients with progressive disease within 2 months prior to blood sampling were excluded from progression analysis. The median serum Ang-2 level in the patient group as a whole was used to divide the patients into two groups (cut-off value was $4756 \mathrm{pg} / \mathrm{ml}$ ). The median follow-up period was 6 months, range 4-8 months. NET patients with serum Ang-2 levels $>4756 \mathrm{pg} / \mathrm{ml}(n=14)$ had a worse prognosis than those with Ang-2 levels $\leq 4756 \mathrm{pg} / \mathrm{ml}$ $(n=22$; Fig. 7). Both groups had similar previous treatments and a similar number of patients on SST analogues (see Fig. 7b).

\section{Discussion}

This is the first study to demonstrate elevated serum Ang-2 levels and Ang-2:Ang-1 ratio in patients with NETs compared healthy controls. Similar findings have been reported in a number of studies looking at serum Ang-2 levels in other cancers including colonic, lung and ovarian cancer (Wong et al. 2000, Shim et al. 2002, Machein et al. 2004, Mi et al. 2006, Park et al. 2007). Furthermore, Ang-2 mRNA levels are elevated in the tumour tissue of a number of other cancers, including human hepatocellular carcinoma, prostate cancer and breast cancer (Ahmad et al. 2001, Etoh et al. 2001, Mitsuhashi et al. 2003, Sfiligoi et al. 2003, Stoeltzing et al. 2003, Lind et al. 2005). Our findings
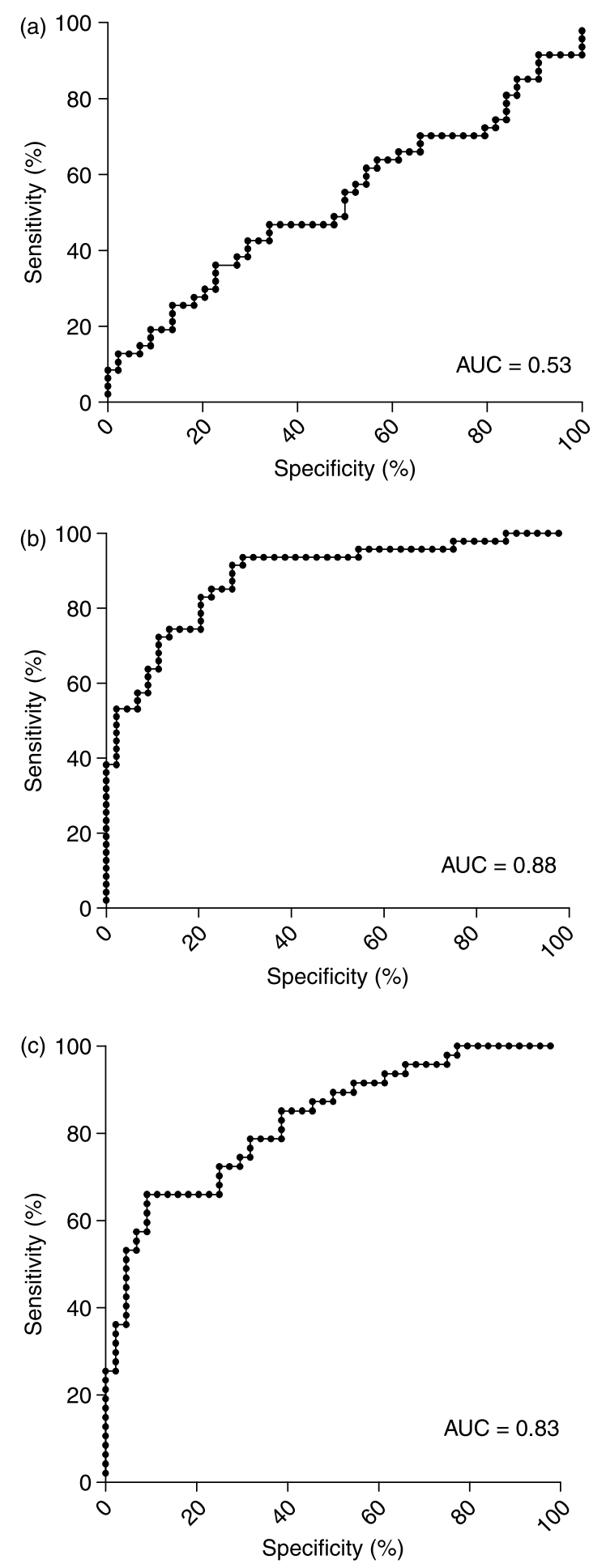

Figure 5 Receiver operator curves for Ang-1 (a), Ang-2 (b) and the ratio of Ang-2 to Ang-1 (c). AUC, area under the curve. 
Table 2 Detection rate (sensitivity), false-positive rates and likelihood ratio for Ang-2 and the ratio of Ang-2 to Ang-1 in NETs

\begin{tabular}{|c|c|c|c|c|c|c|c|c|}
\hline \multirow{2}{*}{$\begin{array}{l}\text { Detection rate } \\
\text { (sensitivity) }\end{array}$} & \multicolumn{4}{|c|}{ Ang-2 } & \multicolumn{4}{|c|}{ Angiopoietin 2:1 ratio } \\
\hline & Cut-off & FPR (\%) & $\mathrm{Cl}(\%)$ & LR & Cut-off & FPR (\%) & $\mathrm{Cl}(\%)$ & LR \\
\hline $70 \%$ & $>3770$ & 11.4 & $3.6-24.6$ & 6.1 & $>0.095$ & 25.0 & $13.2-40.3$ & 2.8 \\
\hline $75 \%$ & $>3565$ & 15.4 & $5.2-27.4$ & 4.9 & $>0.088$ & 31.8 & $18.6-47.6$ & 2.4 \\
\hline $80 \%$ & $>3447$ & 21.5 & $9.8-35.3$ & 3.7 & $>0.074$ & 38.6 & $24.4-55.6$ & 2.1 \\
\hline $85 \%$ & $>3313$ & 22.7 & $11.5-37.8$ & 3.7 & $>0.071$ & 38.4 & $24.4-52.2$ & 2.3 \\
\hline $90 \%$ & $>3172$ & 27.3 & $15.0-42.8$ & 3.3 & $>0.0644$ & 55.6 & $38.8-69.4$ & 1.7 \\
\hline $95 \%$ & $>2447$ & 55.8 & $38.8-69.6$ & 1.7 & $>0.0527$ & 65.9 & $50.1-79.6$ & 1.5 \\
\hline
\end{tabular}

The cut-off values for Ang-2 are in pg/ml. FPR, false-positive rate; Cl, confidence interval; LR, likelihood ratio.

suggest that Ang-2 was superior to Ang-1 for differentiating NET patients from control samples, and distinguishing patients with distant metastasis from those without and appears to increase in relation to increasing disease burden. In addition, our multivariate analysis suggests that high levels of Ang-2 are associated with higher grade tumours.

NETs often progress at a relatively slow rate; however, occasionally they may grow rapidly. During periods of growth or progressive disease, there may well be a shift in proangiogenic factors including Ang-2. This could be a reflection on the biology and natural course of NETs. Interpretation of Ang-1 levels is difficult due to the wide range of values expressed and the complex behaviour of this growth factor. Interestingly, high levels of Ang-1 were not correlated with increased Ang-2 levels. However, the higher ratio of Ang-2 is more commonly associated with high tumour load, i.e. $>50 \%$ liver metastases rather than low-volume disease or patients without metastasis. Our results support the hypothesis that elevation of Ang-2 being an important aspect of tumour angiogenesis.

This study has shown Ang-2 as a marker, with high sensitivity, however, lacking specificity for use as a single marker for NETs since it is raised in a number of cancers and patients with cirrhosis. However, it could potentially have a role as an additional marker for monitoring the development of recurrent disease or distant metastases, although further validation of this marker will be required. Since $\mathrm{CgA}$ lacks sensitivity in cases with low tumour volume (Eriksson et al. 2000), Ang-2 levels could be useful since there was a significant difference in Ang-2 levels in controls and patients without metastatic (i.e. low volume) disease, however, the number of patients with localized disease were small $(n=6)$.

In our study, while Ang-1 did not have a significant prognostic implication, serum Ang-2 was more promising as a potential prognosticator; with levels $>4756 \mathrm{pg} / \mathrm{ml}$ associated with earlier time to disease progression. The exact value for the cut-off will require prospective validation in another data set. Whether the level of serum Ang-2 is also a predictor of survival will require re-evaluation after prolonged follow-up.

Currently, there are no accurate biochemical markers to aid in the diagnosis of progressive disease in patients with NETs. Even though $\mathrm{CgA}$ has been reported to have prognostic value especially in midgut

Table 3 Immunohistochemical staining for Ang-2 performed in nine patients

\begin{tabular}{|c|c|c|c|c|c|}
\hline Primary & Grade & Functional & Biopsy site & Ang-2 (pg/ml) & Staining pattern \\
\hline Midgut & Low grade & Yes & Liver & 6800 & Negative \\
\hline Midgut & Low grade & Yes & Liver & 13708 & $\begin{array}{l}\text { Moderate staining, granular staining } \\
10 \% \text { and strong staining in cyto- } \\
\text { plasm } 1 \% \text { of cells }\end{array}$ \\
\hline Midgut & Low grade & Yes & Liver & 6205 & Negative \\
\hline Pancreas & Low grade & No & Pancreas & 5983 & Negative \\
\hline Pancreatic & Low grade & No & Liver & 6152 & Moderate staining in $1 \%$ cells \\
\hline Pancreas & Low & Yes & Liver & 3815 & Strong positivity in $1 \%$ cells \\
\hline Pancreas & Intermediate & Yes & Liver & 10540 & Strong positivity in $1 \%$ cells \\
\hline Pancreas & Low & No & Liver & 3752 & Strong positivity in $1 \%$ cells \\
\hline Pancreatic & Intermediate & No & Gall bladder/liver & 14846 & $\begin{array}{l}5 \% \text { strong positive in gall bladder } \\
\text { tumour, } 1 \% \text { positive staining in liver }\end{array}$ \\
\hline
\end{tabular}

Table shows site of primary, location and grade of tumour biopsy and staining pattern for Ang-2. 
(a)

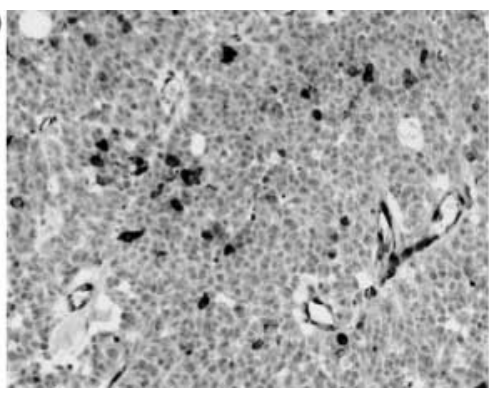

(b)

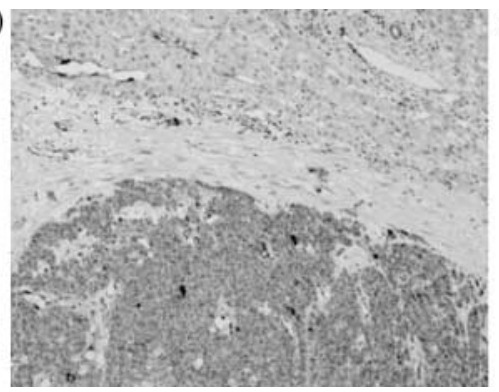

(c)

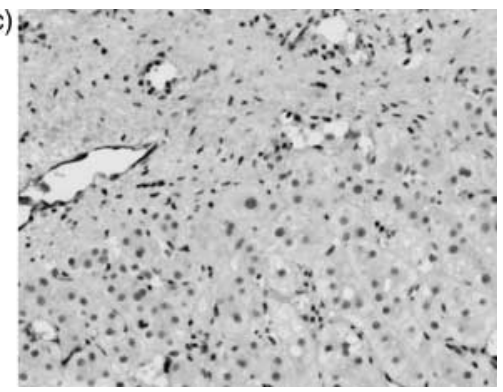

Figure 6 (a) Ang-2 staining in NET invading the gall bladder shows strong Ang-2 positivity in tumour cells and endothelial cells (internal control), $\times 20$ magnification. (b) The top part of the image is normal liver with negative Ang-2 staining and the lower part of the image is liver metastatic NET, with scattered Ang-2 positive staining, $\times 10$ magnification. (c) Normal liver showing only endothelial positive staining for Ang-2, $\times 20$ magnification.

carcinoid tumours (Janson et al. 1997), it is not 100\% sensitive. Therefore, the use of Ang-2 in patients with NET could serve as a marker indicating likelihood of developing progressive disease.

There was no significant difference in Ang-2 levels between patients treated with SST analogues and those not. Since SST analogues are thought to inhibit angiogenesis through a number of pathways, the lack of difference between the groups at first may seem surprising. However, there may be a number of reasons no difference is seen, most importantly is the heterogeneity of the groups. The tumour load of patients on SST analogues is often greater than those not on SST analogue therapies, since these patients all have metastatic tumours and hence syndromic. We have demonstrated that patients with large-volume disease generally had high Ang-2 levels.

We have demonstrated by immunohistochemistry that a minority of NET cells strongly express Ang-2 in contrast to the normal liver. We did not find any clear

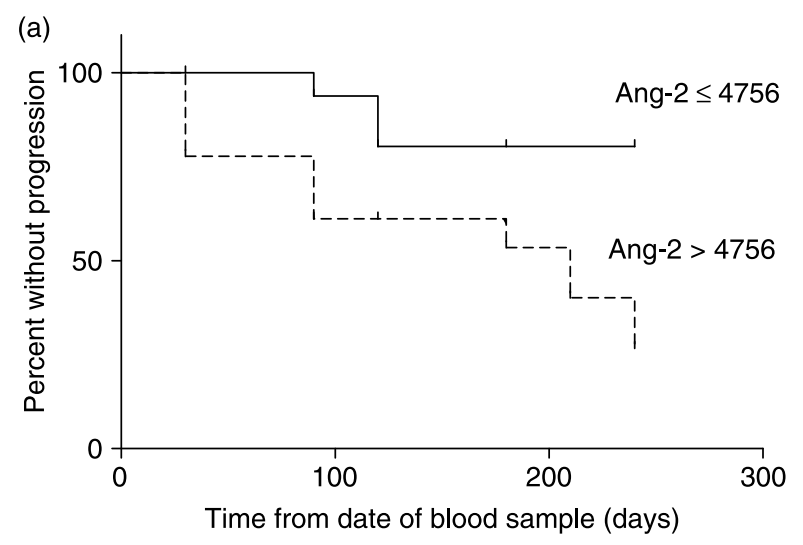

(b)

\begin{tabular}{|l|c|c|}
\hline Therapy & Ang-2 $\leq 4756(n=22)$ & Ang-2 $>4756(n=14)$ \\
\hline Concurrent SST analogues & $14(63.6 \%)$ & $8(57.1 \%)$ \\
\hline Prior therapy & & \\
\hline Chemotherapy & $2(9.1 \%)$ & $3(21.4 \%)$ \\
\hline Interferon & $2(9.1 \%)$ & $0(0 \%)$ \\
\hline Radiotargeted therapy & $5(22.7 \%)$ & $2(14.3 \%)$ \\
\hline Right hepatectomy & $4(18.2 \%)$ & $1(7.1 \%)$ \\
\hline Other surgery & $3(13.6 \%)$ & $5(35.7 \%)$ \\
\hline None & $3(13.6 \%)$ & $1(7.1 \%)$ \\
\hline
\end{tabular}

Figure 7 (a) Kaplan-Meier curves of time to progression of NETs according to Ang-2 levels. Log rank $P=0.03$. (b) Treatments undergone by patients in the two groups during the course of their illness and the number of patients on current somatostatin analogue therapy. 
correlation between tumour staining and serum levels. There are three explanations for this; first, there may be heterogeneity of expression within the tumour, as we have demonstrated clearly in one case, and one small sample may not be representative. Second, the Ang-2 serum level appears to be related to tumour burden. Hence, a small tumour with high Ang-2 expression may result in a lower serum level than a bulky metastatic tumour with a lower Ang-2 expression. Finally, we cannot exclude the possibility that the Ang-2 arises from another source, but its release is associated with tumour.

There were limitations with our study; first, the study incorporated a rather diverse group of patients including patients with NETs with a foregut, midgut and those with unknown primaries. Second, the study group had a number of different previous treatments and some patients had stable disease without any evidence of radiological progression, while others had progressive disease at time of serum collection. This may in part explain the heterogeneity identified with Ang-1 and Ang-2 levels.

In conclusion, this is the first study reporting that serum Ang-2 is elevated in patients with NETS and that it may serve as a useful marker in NETs for monitoring and prognostication. A greater understanding of the role of Ang-1 and -2 in the pathogenesis of NETs may provide an opportunity for the development of effective therapy.

\section{Declaration of interest}

The authors declare that there is conflict of interest that could be perceived as prejudicing the impartiality of the research reported.

\section{Funding}

This research did not receive any specific grant from any funding agency in the public, commercial or not-forprofit sector. G Dancey is funded by Cancer Research UK and the work was supported by the UCL Experimental Cancer Medicine Centre. Part of this work was undertaken at UCLH/UCL who received a proportion of funding from the Department of Health's NIHR Biomedical Research Centres funding scheme.

\section{References}

Ahmad SA, Liu W, Jung YD, Fan F, Wilson M, Reinmuth N, Shaheen RM, Bucana CD \& Ellis LM 2001 The effects of angiopoietin-1 and -2 on tumor growth and angiogenesis in human colon cancer. Cancer Research 61 1255-1259.

Bach F, Uddin FJ \& Burke D 2007 Angiopoietins in malignancy. European Journal of Surgical Oncology 33 7-15.
Caplin ME, Buscombe JR, Hilson AJ, Jones AL, Watkinson AF \& Burroughs AK 1998 Carcinoid tumour. Lancet 352 799-805.

Carmeliet P \& Jain RK 2000 Angiogenesis in cancer and other diseases. Nature 407 249-257.

Davis S, Aldrich TH, Jones PF, Acheson A, Compton DL, Jain V, Ryan TE, Bruno J, Radziejewski C, Maisonpierre PC et al. 1996 Isolation of angiopoietin-1, a ligand for the TIE2 receptor, by secretion-trap expression cloning. Cell 87 1161-1169.

Eriksson B, Oberg K \& Stridsberg M 2000 Tumor markers in neuroendocrine tumors. Digestion 62 33-38.

Eriksson B, Kloppel G, Krenning E, Ahlman H, Plockinger U, Wiedenmann B, Arnold R, Auernhammer C, Korner M, Rindi $\mathrm{G}$ et al. 2008 Consensus guidelines for the management of patients with digestive neuroendocrine tumors - well-differentiated jejunal-ileal tumor/carcinoma. Neuroendocrinology 87 8-19.

Etoh T, Inoue H, Tanaka S, Barnard GF, Kitano S \& Mori M 2001 Angiopoietin-2 is related to tumor angiogenesis in gastric carcinoma: possible in vivo regulation via induction of proteases. Cancer Research 61 2145-2153.

Folkman J \& Hanahan D 1991 Switch to the angiogenic phenotype during tumorigenesis. Princess Takamatsu Symposium 22 339-347.

Folkman J, Watson K, Ingber D \& Hanahan D 1989 Induction of angiogenesis during the transition from hyperplasia to neoplasia. Nature 339 58-61.

de Herder WW 2007 Biochemistry of neuroendocrine tumours. Best Practice \& Research. Clinical Endocrinology \& Metabolism 21 33-41.

Holash J, Wiegand SJ \& Yancopoulos GD 1999 New model of tumor angiogenesis: dynamic balance between vessel regression and growth mediated by angiopoietins and VEGF. Oncogene 18 5356-5362.

Janson ET, Holmberg L, Stridsberg M, Eriksson B, Theodorsson E, Wilander E \& Oberg K 1997 Carcinoid tumors: analysis of prognostic factors and survival in 301 patients from a referral center. Annals of Oncology 8 685-690.

Lee HJ, Cho CH, Hwang SJ, Choi HH, Kim KT, Ahn SY, Kim JH, Oh JL, Lee GM \& Koh GY 2004 Biological characterization of angiopoietin-3 and angiopoietin-4. FASEB Journal 18 1200-1208.

Lind AJ, Wikstrom P, Granfors T, Egevad L, Stattin P \& Bergh A 2005 Angiopoietin 2 expression is related to histological grade, vascular density, metastases, and outcome in prostate cancer. Prostate 62 394-399.

Machein MR, Knedla A, Knoth R, Wagner S, Neuschl E \& Plate KH 2004 Angiopoietin-1 promotes tumor angiogenesis in a rat glioma model. American Journal of Pathology 165 1557-1570.

Maisonpierre PC, Suri C, Jones PF, Bartunkova S, Wiegand SJ, Radziejewski C, Compton D, McClain J, Aldrich TH, Papadopoulos N et al. 1997 Angiopoietin-2, a natural antagonist for Tie 2 that disrupts in vivo angiogenesis. Science 277 55-60. 
Mi J, Sarraf-Yazdi S, Zhang X, Cao Y, Dewhirst MW, Kontos CD, Li CY \& Clary BM 2006 A comparison of antiangiogenic therapies for the prevention of liver metastases. Journal of Surgical Research 131 97-104.

Mitsuhashi N, Shimizu H, Ohtsuka M, Wakabayashi Y, Ito H, Kimura F, Yoshidome H, Kato A, Nukui Y \& Miyazaki M 2003 Angiopoietins and Tie-2 expression in angiogenesis and proliferation of human hepatocellular carcinoma. Hepatology 37 1105-1113.

Modlin IM, Lye KD \& Kidd M 2003 A 5-decade analysis of 13,715 carcinoid tumors. Cancer 97 934-959.

Modlin IM, Oberg K, Chung DC, Jensen RT, de Herder WW, Thakker RV, Caplin M, Delle FG, Kaltsas GA, Krenning EP et al. 2008 Gastroenteropancreatic neuroendocrine tumours. Lancet Oncology 9 61-72.

Oliner J, Min H, Leal J, Yu D, Rao S, You E, Tang X, Kim H, Meyer S, Han SJ et al. 2004 Suppression of angiogenesis and tumor growth by selective inhibition of angiopoietin-2. Cancer Cell 6 507-516.

Park JH, Park KJ, Kim YS, Sheen SS, Lee KS, Lee HN, Oh YJ \& Hwang SC 2007 Serum angiopoietin-2 as a clinical marker for lung cancer. Chest 132 200-206.

Rindi G, Kloppel G, Couvelard A, Komminoth P, Korner M, Lopes JM, McNicol AM, Nilsson O, Perren A, Scarpa A et al. 2007 TNM staging of midgut and hindgut (neuro) endocrine tumors: a consensus proposal including a grading system. Virchows Archiv 451 757-762.

Sfiligoi C, de LA, Cascone I, Sorbello V, Fuso L, Ponzone R, Biglia N, Audero E, Arisio R, Bussolino F et al. 2003 Angiopoietin-2 expression in breast cancer correlates with lymph node invasion and short survival. International Journal of Cancer 103 466-474.

Shim WS, Teh M, Bapna A, Kim I, Koh GY, Mack PO \& Ge R 2002 Angiopoietin 1 promotes tumor angiogenesis and tumor vessel plasticity of human cervical cancer in mice. Experimental Cell Research 279 299-309.
Stoeltzing O, Ahmad SA, Liu W, McCarty MF, Wey JS, Parikh AA, Fan F, Reinmuth N, Kawaguchi M, Bucana CD et al. 2003 Angiopoietin-1 inhibits vascular permeability, angiogenesis, and growth of hepatic colon cancer tumors. Cancer Research 63 3370-3377.

Suri C, Jones PF, Patan S, Bartunkova S, Maisonpierre PC, Davis S, Sato TN \& Yancopoulos GD 1996 Requisite role of angiopoietin-1, a ligand for the TIE2 receptor, during embryonic angiogenesis. Cell 87 1171-1180.

Tait CR \& Jones PF 2004 Angiopoietins in tumours: the angiogenic switch. Journal of Pathology 204 1-10.

Tanaka S, Sugimachi K, Yamashita Y, Shirabe K, Shimada M, Wands JR \& Sugimachi K 2003 Angiogenic switch as a molecular target of malignant tumors. Journal of Gastroenterology 38 93-97.

Therasse P, Arbuck SG, Eisenhauer EA, Wanders J, Kaplan RS, Rubinstein L, Verweij J, Van GM, van Oosterom AT, Christian MC et al. 2000 New guidelines to evaluate the response to treatment in solid tumors. European Organization for Research and Treatment of Cancer, National Cancer Institute of the United States, National Cancer Institute of Canada. Journal of the National Cancer Institute 92 205-216.

Wang J, Wu K, Zhang D, Tang H, Xie H, Hong L, Pan Y, Lan M, Hu S, Ning X et al. 2005 Expressions and clinical significances of angiopoietin-1, -2 and Tie2 in human gastric cancer. Biochemical and Biophysical Research Communications 337 386-393.

Wong MP, Chan SY, Fu KH, Leung SY, Cheung N, Yuen ST \& Chung LP 2000 The angiopoietins, tie2 and vascular endothelial growth factor are differentially expressed in the transformation of normal lung to non-small cell lung carcinomas. Lung Cancer 29 11-22.

Yu Q \& Stamenkovic I 2001 Angiopoietin-2 is implicated in the regulation of tumor angiogenesis. American Journal of Pathology 158 563-570. 